\title{
On the genus Chrysometa (Araneae, Tetragnathidae) in south Brazil
}

\author{
Thiago B. Massanti', Rodney R. Cavichioli' \& Ricardo Ott ${ }^{2}$
}

1. Universidade Federal do Paraná, Departamento de Zoologia, Mailbox 19020, 81531-980, Curitiba, PR, Brazil. (tbrobio@gmail.com, cavich@ufpr.br)
2. Museu de Ciências Naturais, Fundação Zoobotânica do Rio Grande do Sul, Rua Dr. Salvador França, 1427, $90690-000$ Porto Alegre, RS, Brazil.

Received 05 July 2017

Accepted 30 November 2017

Published 08 January 2018

DOI: $10.1590 / 1678-4766 e 2018001$

ABSTRACT. The male of Chrysometa cambara Levi, 1986 is considered as belonging to C. itaimba Levi, 1986. The female of C. aramba Levi, 1986 and the male newly assigned to $C$. cambara Levi 1986 are described for the first time. Reviewed records for $C$. aramba, $C$. cambara, $C$. itaimba, $C$. boraceia Levi, 1986 and C. ludibunda (Keyserling, 1893) are presented.

KEYWORDS. Araneoidea, Metainae, geographic distribution, Systematics.

RESUMO. Sobre o gênero Chrysometa (Araneae, Tetragnathidae) no sul do Brasil. O macho de C. cambara Levi, 1986 é considerado como pertencente à $C$. itaimba Levi, 1986. A fêmea de Chrysometa aramba Levi, 1986 e o novo macho atribuído à $C$. cambara Levi, 1986 são descritos pela primeira vez. Registros atualizados de C. aramba, C. cambara, C. itaimba, C. boraceia Levi, 1986 e C. ludibunda (Keyserling, 1893 ) são apresentados.

PALAVRAS-CHAVE. Araneoidea, Metainae, distribuição geográfica, Sistemática.

Tetragnathidae Menge, 1866 is a cosmopolitan spider family belonging to the Araneoidea (ÁlLVAREZ-PADILla et al., 2009). Nowadays species of the family are distributed in 49 genera and 994 species and the highest diversity of the family is found in tropical and subtropical regions (WORLD SPIDER CATAlog, 2017). Tetragnathidae differs from other araneoids by the following characters: simple male pedipalp, conductor and embolus coiling together, characteristic spinneret spigot anatomy and particular web building behaviors (ÁLVAREZPAdilla \& Hormiga, 2011).

Chrysometa was proposed by Simon (1894) to include the species described as Tetragnatha tenuipes Keyserling, 1864. Specimens of the genus differ from other tetragnathids by presenting femurs IV lacking trichobothria combined to abdomens with silver or white spots; males presents a cymbial apophysis and a distinctive terminal apophysis; females present a flat epigyne usually without projections and fertilization ducts often more sclerotized than connecting ducts (LeVI, 1986; NoGUEIRA et al., 2011). Apart from smaller size, males of the genus usually have longer legs than females (LEVI, 1986).

LEVI (1986) published the only taxonomic review of the group illustrating 127 of the 139 species of Chrysometa, many of them known only by one of the sexes (WorLD SPIDER Catalog, 2017). However, the distributional data of Chrysometa is still largely unknown and, since many species of the genera are described based on single specimens, it can be expected that several species are yet to be discovered (Álvarez-Padilla, 2007; LeVI, 1986; NogueIra et al., 2011). Based on the examination of a large amount of males and females recently collected together in a ecological survey at Paraná State, in southern Brazil, and the examination of material of the arachnological collection of the Museu de Ciências Naturais da Fundação Zoobotânica do Rio Grande do Sul (MCN), we conclude that the male described by LEVI (1986) as belonging C. cambara Levi, 1986 actually belongs to $C$. itaimba Levi, 1986. Herein we also describe the female of Chrysometa aramba Levi, 1986 and the new male assigned to $C$. cambara. Geographical distribution data based on new MCN records are presented for the above cited species and also for $C$. boraceia Levi, 1986 and $C$. ludibunda (Keyserling, 1893), including recent collection data and reviewed records from Rio Grande do Sul State presented by BucKuP et al. (2010).

\section{MATERIAL AND METHODS}

All specimens examined are deposited at the Arachnological collection of Museu de Ciências Naturais da Fundação Zoobotânica do Rio Grande do Sul, Porto Alegre, Brazil (MCN; R. Ott). Other institutions referred: American Museum of Natural History, New York, USA (AMNH), 
Museum of Comparative Zoology, Harvard University, Cambridge, USA (MCZ), Museu de Zoologia, Universidade de São Paulo, São Paulo, Brazil (MZSP).

The left male palps were detached for examination; epigynes were also removed from the abdomen and examined immersed in clove oil. Incident light images were taken through a stereomicroscope with attached camera and processed with Helicon Focus multi-range program. Transmitted light images of cleared female epigynes were taken with a compound microscope using a digital camera and also processed with Helicon Focus (KozuB et al., 2012). Drawings were made using printed images as models and compound microscope and stereomicroscope as details confirmation tools.

Nomenclature is based on LEVI (1986). The asterisk symbol "*" after locality means "New record". All measures are in millimeters.

\section{TAXONOMY}

\section{Chrysometa aramba Levi, 1986}

(Figs 1-14, 38)

Chrysometa aramba LEvI, 1986:144, figs. 231-233. Male holotype from Cambará do Sul, Rio Grande do Sul, Brazil, 9.I.1976, A. A. Lise leg., deposited in MCN 3348 (examined).

Other material examined. BRAZIL, Rio Grande do Sul: Canela*, $q$, 28.IX.2000, A. L. H. Silva leg. (MCN 33360); São Francisco de Paula, ô, 25.XI.1998, A. L. H. Silva leg. (MCN 30960); ㅇ, 1.II.1999, A. L. H. Silva leg. (MCN 30883); Candelaria*,, , 5.II.2001, R. Ott leg. (MCN 33619).

Diagnosis. This species is very close to $C$. boquete Levi, 1986 by having a discrete conical apophysis on the dorso-proximal region of the cymbium with no ectobasal or ectomedian process in males (Figs 4, 5; see also LeVI, 1986:138, 139, figs 190, 191) and by the presence of a large knob-like epigynal septum and reniform shaped spermatheca with wrapped spermatic ducts in females (Figs 9-14; see also LeVI, 1986:138, 139, figs 185-188). Males of C. aramba (Figs 1-5) can be also distinguished by the palp bearing a flattened, oval shaped paracymbial apophysis, with bulged prolateral border and smooth lower end (Figs 4, 5), which is corniculate in $C$. boquete. Females can be further distinguished by the almost as wide as long trapezoidal shaped median knob-like septum (Figs 9, 10), which is wider than long in C. boquete.

Description. Female (MCN 33360). Carapace orange (Fig. 6). Chelicerae brown (Figs 7, 8). Sternum, endites and labium brown darker on borders (Fig. 7). Legs I and II orange with darker marks on distal regions of femur, patella and tibia regions (Figs 6-8). Opisthosoma oval; dorsally with two longitudinal brown spots on anterior region; posteriorly chevron like pattern formed by brown transverse bars (Fig. 6); lateral of abdomen with silver spots in the all extension (Fig. 8). Ventral region dark gray, with two whitish stripes (Fig. 7). Measurements (mm): AME 0.063; ALE 0.042; PME
0.084; PLE 0.042; AME - AME 0.042; AME - ALE 0.063; PME - PME 0.084; Total Length 2.90; Carapace 1.18 long, wide 0.97; Femur I 1.73; Patella + Tibia I 2.01; Metatarsus I 1.87; Tarsus I 0.61; Patella + Tibia II 1.37; Patella + Tibia III 0.74; Patella + Tibia IV 1.05.

Male. See Levi, 1986:143, 144, figs. 231-233.

Variation. Total length: males, $2.70 *-2.91\left({ }^{*} \mathrm{LEVI}\right.$, 1986:144 data); females, 2.90-3.43.

Distribution. Known only for Rio Grande do Sul State, Brazil.

\section{Chrysometa cambara Levi, 1986}

(Figs 15-27, 38)

Chrysometa cambara LevI, 1986:192, figs 618-623. Female holotype from Itaimbezinho, Cambará do Sul, Rio Grande do Sul, Brazil, 5.I.1985, A. Lise leg., deposited in MCN 12795 (examined). Paratypes: female, same data of holotype (MCN 12800; examined); female, Teresópolis (1800 m), Rio de Janeiro, Brazil, 15.III.1946, H. Sick leg. and female, (open stone cave) same data above (AMNH, both not examined); male paratype, Porto Alegre, 15.VIII.1976, P. A. Buckup leg. (MCN 4546, examined; see note below). Only the female holotype and female paratypes; not the male paratype (misidentification, male same as $C$. itaimba Levi, 1986).

Note. Levi (1986:192) stated that the males and females described as $C$. cambara does not necessarily belong together. MCN records indicated an additional female "paratype" (ex-MCN 12784; same data of holotype) which should be deposited at MCZ; however this specimen is not indicated at the original description.

Other material examined. BRAZIL, Paraná: Irati*, Ô, 20.II.2012 (MCN 52197); ${ }^{7}$, 5.III.2012 (MCN 52195); Tijucas do Sul (Serra do Cabral*), §., 30.I.2012 (MCN 52196); ㅇ, 2.IV.2012 (MCN 52190); , 5.I.2012 (MCN 52191); ㅇ, 30.I.2012 (MCN 52192); , 5.II.2012 (MCN 52193); ․, 30.I.2012 (MCN 52194); 2ð, 30.I.2012 (MCN 52230), all J. Ricetti leg. Rio Grande do Sul: Cambará do Sul, 2o, 25.XI.1993, L. A. Moura leg. (MCN 24259); ㅇ, 19-21.XII.1994, E. H. Buckup leg. (MCN 25988); 3 ㅇ, 19-21.XII.1994, E. H. Buckup leg. (MCN 26050); São Francisco de Paula,, , X.2001, R. Baldissera leg. (MCN 35223); $\widehat{\text {, }}$, 29.I.2006, L. A. Moura leg. (MCN 40356).

Diagnosis. Females (Figs 22-27) of the species can be easily recognized by the shape of the epigynum, with the septum bearing two heavily sclerotized lobes (Figs 25-27; see also LeVI, 1986:192, 193, figs 620,621). Males of the species (Figs 15-21) are close to C. calima Levi, 1986 and C. bolivia Levi, 1986 by the general triangular shape of the bulb, bearing a broad, coiled and long embolus (Figs 19-21, see also LEVI, 1986:202, 203, figs 708, 709); they can be distinguished from those of both species by the shape of the paracymbial apophysis bearing a long and concave lower portion (Figs 18-21).

Description. Male (MCN 52197). Carapace yellow. Lateral view with brown mark on the anterior region, starting at the high of the chelicerae. Chelicerae dark brown with two serrated carina on lateral of each chelicerae. Eye region dark; legs yellow with brown marks near to the articulations (Figs 15-17). Opistossoma oval shaped, gray, anteriorly with median silver spots and lateral dark spots; posteriorly with median dark irregularly spoted transverse pattern and lateral 


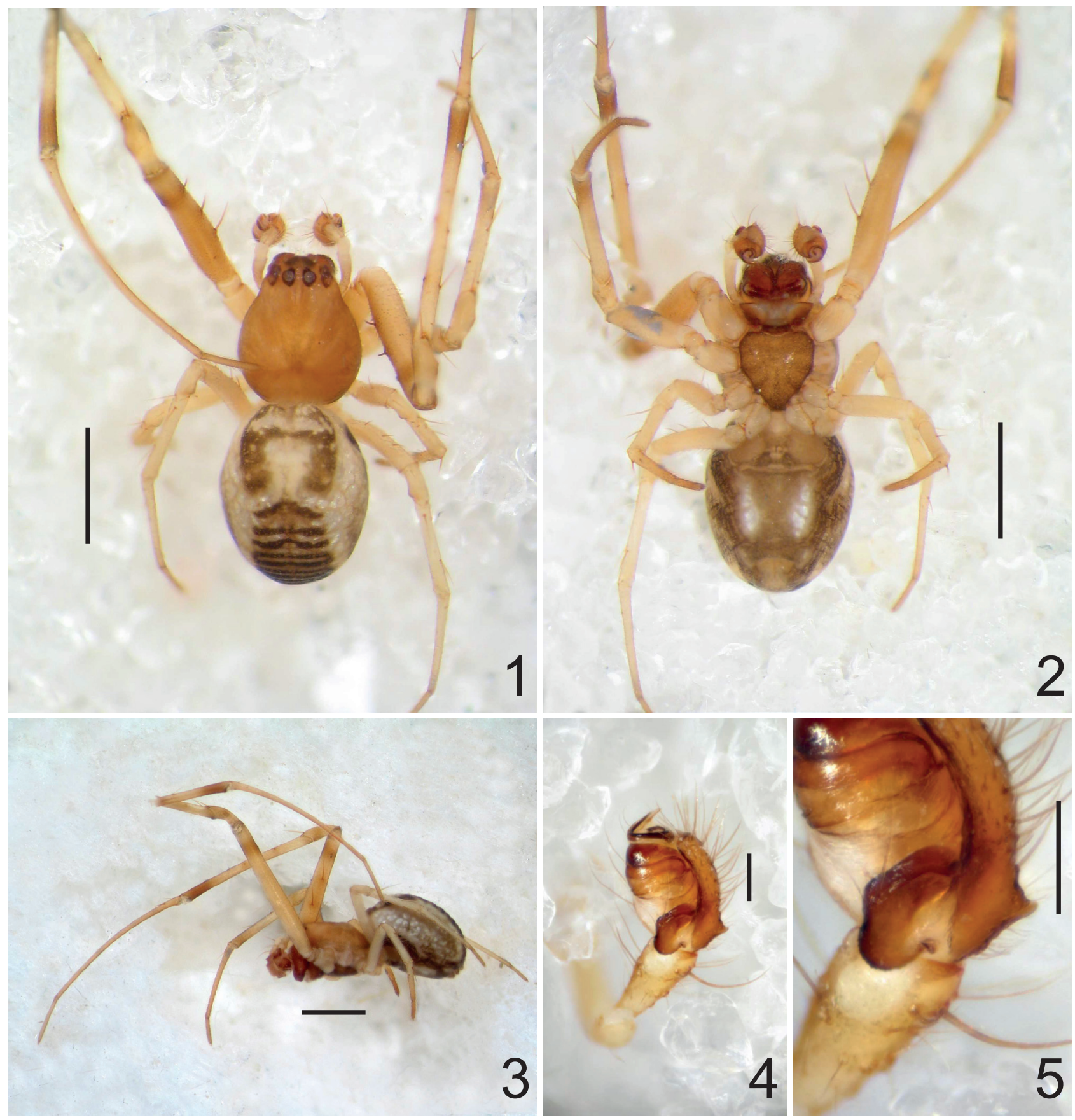

Figs 1-5. Chrysometa aramba Levi, 1986. 1-3 male holotype MCN 3348, 4-5 male MCN 30960. 1, dorsal; 2, ventral; 3, lateral; 4, palp (retrolateral view); 5, paracymbium (retrolateral view). Scale bars: Figs 1-3, $1 \mathrm{~mm}$; Figs 4, 5, $0.1 \mathrm{~mm}$.

silver spots (Fig. 15). Venter greyish with dark grey borders with a central "X" shaped portion of same color and some 3-4 silver spots. Measurements (mm): AME 0.130; ALE 0.156; PME 0.182; PLE 0.104; AME - AME 0.104; AME - ALE 0.104; PME - PME 0.156; Total Length 6.55; Carapace 3.56 long, 2.60 wide; Femur I 7.24; Patella + Tibia I 8.67;
Metatarsus I 5.814; Tarsus I 1.94; Patella + Tibia II 1.63; Patella + Tibia III 2.34; Patella + Tibia IV 3.64.

Female. See LevI, 1986:192-193; figs. 618-621.

Variation. Total length: males, 5.46-6.55; females, 6.24-7.45.

Distribution. South and Southeast of Brazil. 


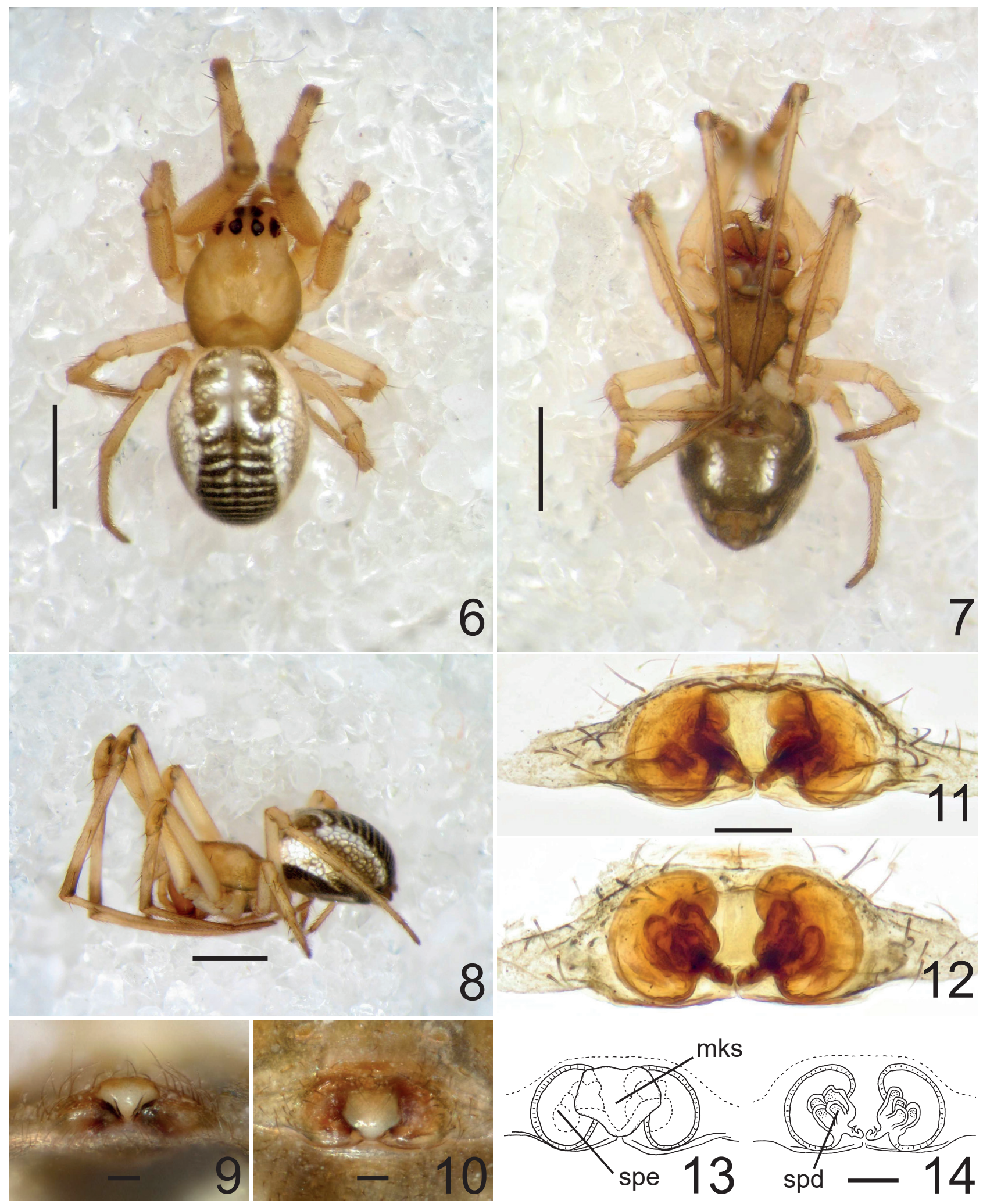

Figs 6-14. Chrysometa aramba Levi, 1986. 6-8 female MCN 33619, 9-14 female MCN 30833. 6, dorsal; 7, ventral; 8, lateral; 9, epigyne (posterior region); 10, epigyne (ventral view); 11, epigyne (ventral view clearead); 12, epigyne (dorsal view clearead); 13, epigyne (ventral view); 14, epigyne (dorsal view) (mks, median knob-like septum; spd, spermatic duct; spe, spermathecae). Scale bars: Figs 6-8, 1 mm; Figs 9-14, 0.1 mm. 


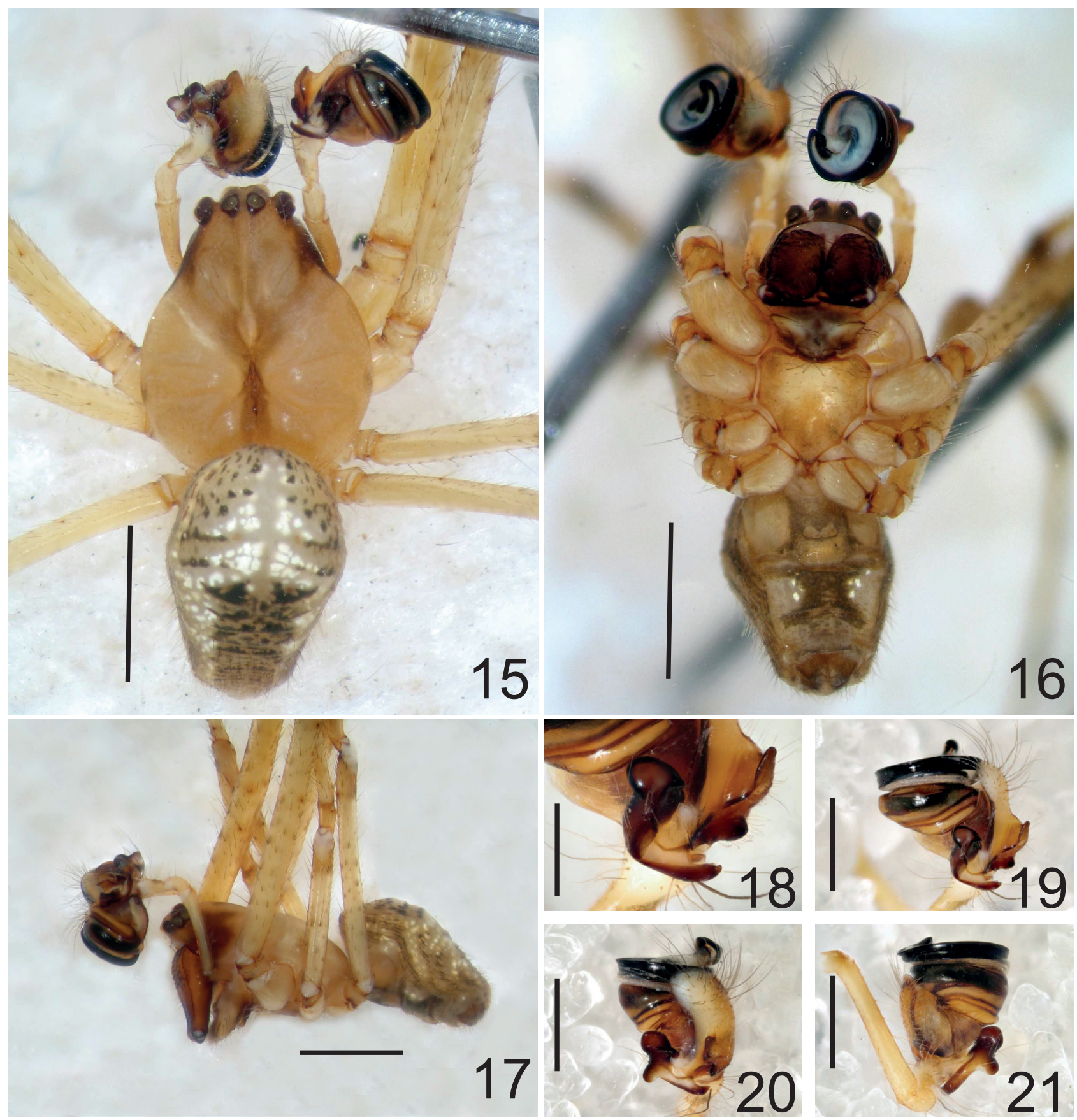

Figs 15-21. Chrysometa cambara Levi, 1986. 15-17 male MCN 52197; 18-21 male MCN 52195. 15, dorsal; 16, ventral; 17, lateral view; 18, paracymbium (ventral view); 19, palp (ventral view); 20, palp (retrolateral view); 21, palp (prolateral view). Scale bars: Figs 15-17, 2 mm; Fig. 18, 0.1 mm; Figs $19-21,0.2 \mathrm{~mm}$.

\section{Chrysometa itaimba Levi, 1986}

(Figs 28-38)

Chrysometa itaimba Levi, 1986:178, figs. 519-522. Female holotype from Itaimbezinho, Cambará do Sul, Rio Grande do Sul, Brazil, 5.I.1985, A. Lise leg, deposited in MCN 12796 (examined). Paratype: female, Campos do Jordão, São Paulo, Brazil, 3.I.1948, F. Lane leg. (MZSP, not examined)
Chrysometa cambara LEVI, 1986:192, figs. 622, 623. Only male paratype of C. cambara, Porto Alegre*, 15.VIII.1976, P. A. Buckup (MCN 4546, misidentification; examined).

Other material examined. BRAZIL, Paraná: Rio Branco do Sul*, ðิ, 16.IV.1987, A. D. Brescovit leg. (MCN 17143); Campina Grande do Sul*, ९, 22.X.2012 (MCN 52226); Tijucas do Sul*, 2ð, 7.V.2012 (MCN 52203, MCN 52230); $\hat{\jmath}, 3$ q 8.V.2012 (MCN 52201); $\hat{\jmath}, 8 . V .2012$ (MCN

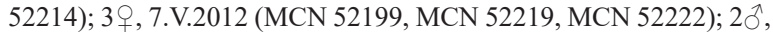


7.V.2012 (MCN 52224); Tijucas do Sul (Serra do Cabral*), ô, 2.IV.2012 (MCN 52225); ð̊, 2ᄋ, 2.IV.2012 (MCN 52227); 2ᄋ, 30.I.2012 (MCN 52215, 52212); ㅇ, 2.IV.2012 (MCN 52200); ㅇ, 2.IV.2012 (MCN 52211); ㅇ, 2.IV.2012 (MCN 52204); Tijucas do Sul (Tabatinga*), วิ), + , 11.V.2012 (MCN 52221); + , 11.V.2012 (MCN 52229); São João do Triunfo*, 2q, 31.III.2012 (MCN 52202, MCN 52228); Fazenda Rio Grande*, ค, 28.XI.2010 (MCN 52220); ठૈ, 19.XII.2010 (MCN

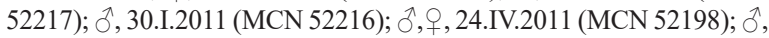
24.IV.2011 (MCN 52208); ภ̂, ㅇ, 1.V.2011 (MCN 52206); ㅇ, 1.V.2011

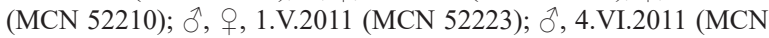
52218); , 6.VIII.2011 (MCN 52207); ðे, 13.VIII.2011 (MCN 52213); , 27.VIII.2011 (MCN 52209); 今̃, 4.I.2012 (MCN 52205); all J. Ricetti leg. Santa Catarina: Porto Belo*,, 14.II.1990, A. D. Brescovit leg. (MCN 19494). Rio Grande do Sul: Cambará do Sul,, , 26.XI.1993, A. B. Bonaldo leg. (MCN 24565); + , 19-21.XII.1994, A. F. Franceschini leg. (MCN 25949); $2 \widehat{\text { }}$, L. A. Moura leg. (MCN 25411); São Francisco de Paula, Ô, 11-12.X.2008, R. B. Moura leg. (MCN 45751); Torres, +, 10-14.I.2005, R. Ott et al. leg. (MCN 38650); Farroupilha, + , 29.IX.1978, H. Bischoff leg. (MCN 8282); Viamão, + , 25.VII.1985, A. A. Lise leg. (MCN 13375); đ̃, 31.V.2000, A. B. Bonaldo leg. (MCN 32896); ô, 30.V.2000, A. B. Bonaldo leg. (MCN 33164); Tavares, + , 22.I.1992, N. Silveira leg. (MCN 21996). Triunfo, Oૈ, 12.I.1989, M. A. L. Marques leg. (MCN 18053); + , 17.IX.1993, A. B. Bonaldo leg. (MCN 23990); ㅇ, 08.I.1997, M. A. L. Marques leg. (MCN 28221); ô, 24.XI.1999, M. A. L. Marques leg. (MCN 31636); ㅇ, 05.VII.2000, E. H. Buckup leg. (MCN 32510); 2 , 05.II.2003, R. Ott leg. (MCN 35192); ㅇ, 29, 30.IV.2003, R. Ott leg. (MCN 35733); §̂, 21.X.2003, R. Ott \& L. Podgaiski leg. (MCN 36445); ค, 28.V.2007, A. Barcellos leg. (MCN 43402); ㅇ, 09.VII.2008, A. Barcellos et al. leg. (MCN 44167);

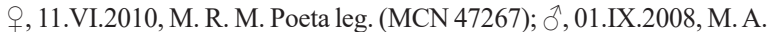
L. Marques leg. (MCN 44342); 今े, 16.IX.2010, E. N. L. Rodrigues leg. (MCN 47372); ; , 16.IX.2010, P. E. S. Rodrigues leg. (MCN 47407); ô, 13.IX.2011, P. E. S. Rodrigues leg. (MCN 48095).

Diagnosis. Females are close to C. nigrovittata (Keyserling, 1893) by the general shape of epigyne (Figs 36-37); they can be distinguished by the shape of the septum, with rounded anterior borders (see LEVI, 1986:181, figs. 519-526). Males are close to C. ramon Levi, 1986 and $C$. columbicola Levi, 1986 by the general shape of bulb and paracymbium (see LEVI, 1986:193, figs 622-627); they can be distinguished from these species by the shape of the conical retrolateral portion of the paracymbium and also by the shape of the cymbial processes with a long and ventrally concave distal process and a conical proximal one (Figs 31, 32).

Description. See Levi, 1986:178, 181, figs. 519-522 for female and LEvi, 1986:192, 193, figs 622, 623 for the male (paratype of C. cambara).

Remarks. LeVI (1986) stated the possibility that male and female originally described for C. cambara does not belong together. The male designed by Levi as paratype was herein identified to belonging to Chrysometa itaimba Levi, 1986, the identification of males of this species is supported by the similarity in male and female sizes and on the coloration pattern between male and female specimens (Figs 28-30, 33-35) and also by the fact that in many samples males and females were collected together.

Variation. Total lenght: males, 3.59-5.20; females, 3.80-5.96. Note: LEVI, 1986:192 states 3.80 total length for the male paratype originally described for C. cambara. This size matches the variation above registered for C. itaimba. All measured males and females of $C$. cambara presents a larger size range (respectively 5.46-6.55 and 6.24-7.45).

Distribution. South and Southeast of Brazil.

\section{Additional new records of south Brazilian Chrysometa species}

\section{Chrysometa boraceia Levi, 1986}

(Fig. 39)

Chrysometa boraceia LevI, 1986:186, figs. 568-575. Female holotype from Boracéia (Salesópolis), São Paulo, Brazil, deposited in MZSP (not examined). Paratypes: female, same data of holotype; four females, Pico da Tijuca, Rio de Janeiro, Brazil, 17.IV.1965, H. Levi leg. (MCZ, not examined); three females, Sumaré, Rio de Janeiro, II.1946, H. Sick leg. (AMNH, not examined); male, Teresópolis, Rio de Janeiro, Brazil, XI.1945 and five females, same loc., III.1946, all H. Sick leg (AMNH, not examined); two males, Boracéia, São Paulo, Brazil, 22-23. II.1961, P. de Biasi leg. (MZSP, not examined); male, same loc. above, XII.1949, Filho leg. (MZSP, not examined); female, same loc. above, X. 1963, Oliveira, P. Wygodzinsky leg. (AMNH, not examined); female, Canela, Rio Grande do Sul, Brazil, 11.V.1974, A. A. Lise leg. (MCN 2133, examined); female, São Francisco de Paula, Rio Grande do Sul, Brazil, A. A. Lise leg. (MCN 9553, examined); female, Montenegro, Rio Grande do Sul, Brazil (MCN 6755, examined).

Other material examined. BRAZIL, Paraná: Campina Grande do Sul*,, , 09.V.2012 (MCN 55072); ㅇ, 09.X.2012 (MCN 55073); ㅇ, 14.X.2012 (MCN 55074); ㅇ, 19.X.2012 (MCN 55075); 2へ, 22.X.2012 (MCN 55076); 우, 27.X.2012 (MCN 55077); 2ㅇ, 03.XII.2012 (MCN 55078). Irati, 20.II.2012 (MCN 55052); 3ㅇ, 20.II.2012 (MCN 55053); 2 우, 20.II.2012 (MCN 55054); 3 ㅇ, 05.III.2012 (MCN 55055, MCN 55056, MCN 55058); $\AA, 2$, 05. III.2012 (MCN 55057); 2 ㅇ, 05.III.2012 (MCN 55059); 4, 07.IV.2012 (MCN 55063, MCN 55064, MCN 55065, MCN 55066); Capitão Leônidas Marques*,, , 20-28.III.1993, A. B. Bonaldo leg. (MCN 23296); Tijucas do Sul (Serra do Cabral*), ô, 30.I.2012 (MCN 55045); 2 , 30.I.2012 (MCN 55046); 29, 30.I.2012 (MCN 55047); ðै, 05.II.2012 (MCN 55048); 4ð̂, 30.I-05.II.2012 (MCN 55082, MCN 55085, MCN 55089, MCN 55090); 2 9, 30.I-05.II.2012 (MCN 55083); 2 , , 30.I-05.II.2012 (MCN 55084); 2 , 30.I-05.II.2012 (MCN 55086); 2 , , 30.I-05.II.2012 (MCN 55087); 2 , 30.I-05.II.2012 (MCN 55088); Tijucas do Sul*, ô, 07.V.2012 (MCN 55067); ㅇ, 08.V.2012 (MCN 55068); Tijucas do Sul (Tabatinga*), đ̂, 10.V.2012 (MCN 55069); 2 , 11.V.2012 (MCN 55070, MCN 55071). São João do Triunfo*, 4, 01.IV.2012 (MCN 55060, MCN 55061, MCN 55062, MCN 55091); Fazenda Rio Grande*, $2 \hat{\jmath}$, 19.XII.2010 (MCN 55033); $\AA$, 30.I.2011 (MCN 55034); $\hat{\jmath}$, ๆ, 05.III.2011 (MCN 55035); 3 \%, 24.IV.2011 (MCN 55036, MCN 55037, MCN 55038); ㅇ, 13.VIII.2011 (MCN 55039); 2 ㅇ, 27.VIII.2011 (MCN 55040, MCN 55041); 2 9, 28.I.2012 (MCN 55042); 29, 28.I.2012 (MCN 55043, MCN 55044); 20 , 18.II.2012 (MCN 55049, MCN 55051); 2 , , 18.II.2012 (MCN 55050); 2 , , 17.XII.2012 (MCN 55079, MCN 55081); ðૈ, ㅇ, 17.XII.2012 (MCN 55080). All above J. Ricetti leg. unless indicated differently. Santa Catarina: Concórdia*, + , 30.I.1996, A. B. Bonaldo leg. (MCN 27264). Rio Grande do Sul: Derrubadas, $9,19-22 . X .2004$, R. Ott et al. leg. (MCN 38702); Bom Jesus, đ̂, 28-31.III.1998, C. Duckett leg. (MCN 29295); Cambará do Sul, ㅇ, 26.XI.1993, A. B. Bonaldo leg. (MCN 24566); 2ㅇ, 19-21. XII.1994, E. H. Buckup leg. (MCN 25989); São Francisco de Paula, ô, 19-22.III.1998, L. A. Moura leg. (MCN 29221). Canela, ㅇ, 23-25. XI.1998, A. F. Franceschini leg. (MCN 29850); ㅇ, 27.IX.2000, E. H. Buckup leg. (MCN 33318); Maquiné*, 12.XII.2007, L. Moura \& R. Moraes leg. (MCN 43775); Viamão, , 29.V.1988, A. B. Bonaldo leg. (MCN 17768); 2 + , 30.V.2000, A. B. Bonaldo leg. (MCN 33165); Porto Alegre, ô, 17.IX.2003, R. Ott, I. Heydrich \& A. Barcellos leg. (MCN 36102); Triunfo, ô, 2 \% , 05.II.2003, R. Ott leg. (MCN 35191); ㅇ, 29-30. IV.2003, R. Ott leg. (MCN 35723); Charqueadas, ग̃, 25.IX.2003, R. Ott leg. (MCN 36435); Dom Feliciano, 2 , 18-19.III.2000, A. B. Bonaldo leg. (MCN 32178); Cristal*, ㅇ, 23.III.2009, E. N. L. Rodrigues \& P. E. S. Rodrigues leg. (MCN 53782); ㅇ, 14.III.2008, E. N. L. Rodrigues leg. (MCN 48829); Pelotas, ô, 01-03.V.1997, L. A. Moura leg. (MCN 28358). Arroio Grande*, , , 18.IV.2009, E. N. L. Rodrigues \& P. E. S. Rodrigues leg. (MCN 54969); ㅇ, 13.VI.2009, E. N. L. Rodrigues \& P.E.S. Rodrigues leg. (MCN 55007). 


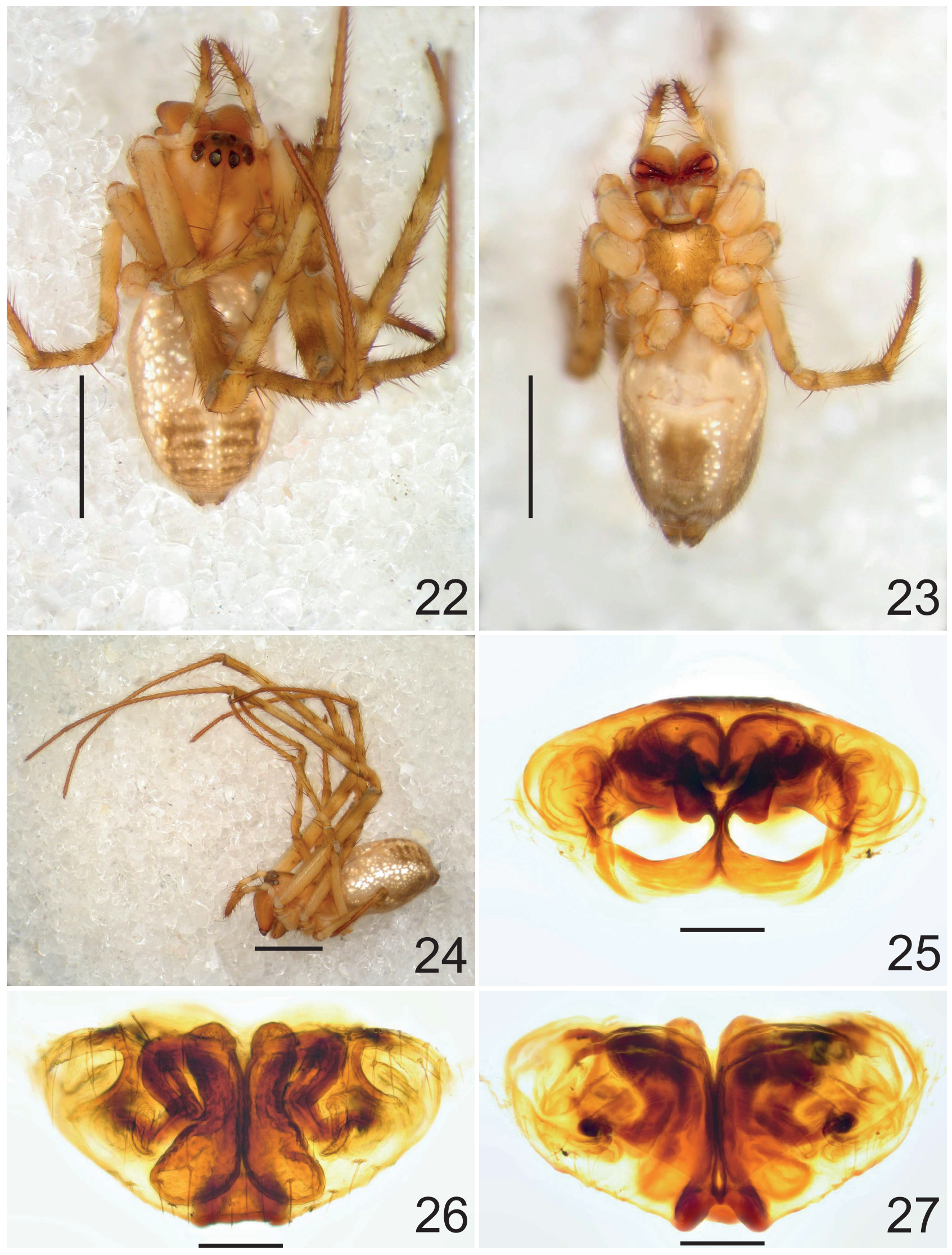

Figs 22-27. Chrysometa cambara Levi, 1986. Female holotype MCN 12795. 22, dorsal; 23, ventral; 24, lateral view; 25, epigyne (posterior view cleared); 26, epigyne (ventral view cleared); 27, epigyne (dorsal view cleared). Scale bars: Figs 22-24, 2 mm; Figs 25-27, $0.1 \mathrm{~mm}$. 


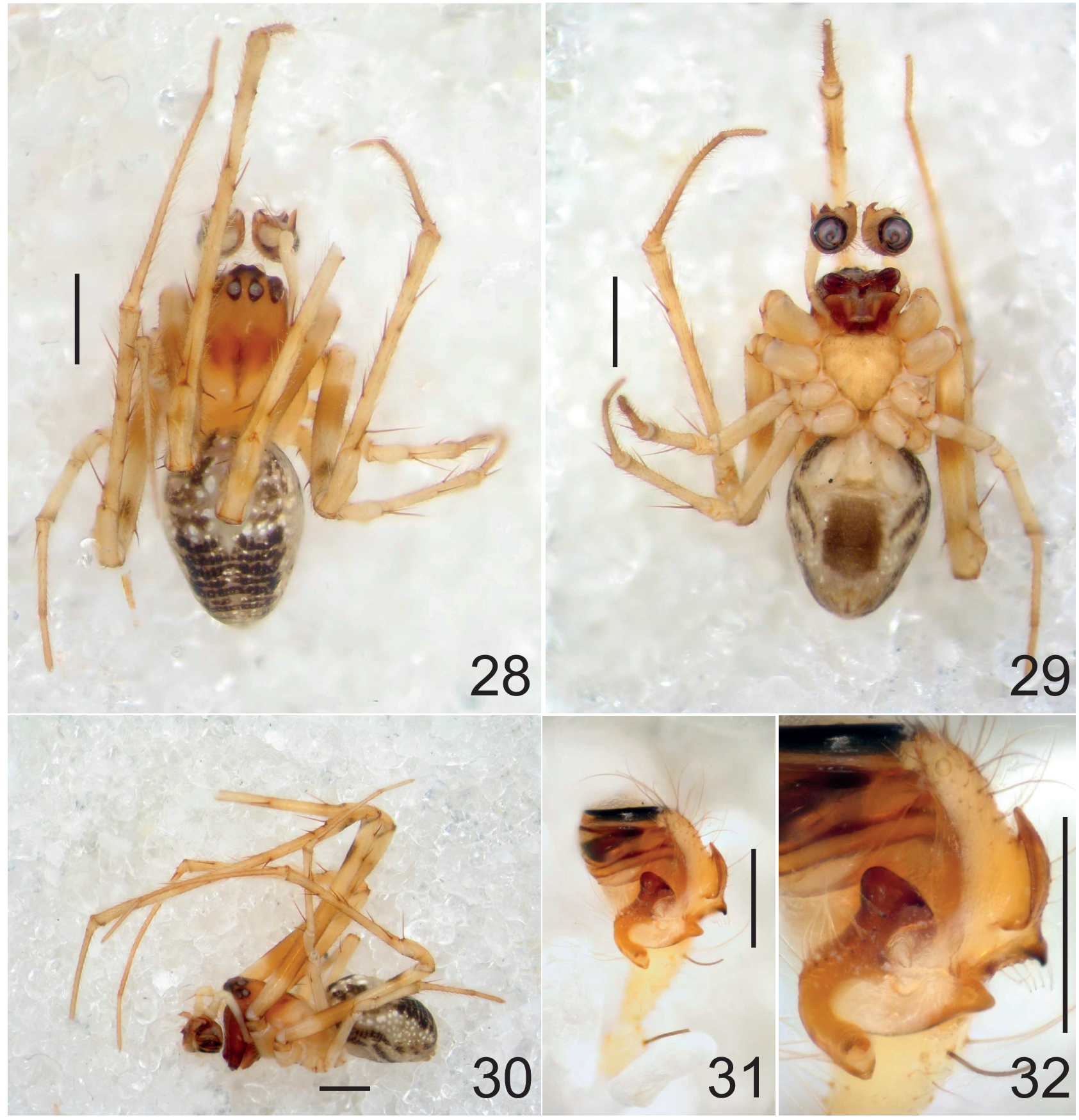

Figs 28-32. Chrysometa itaimba Levi, 1986. 28-30 male MCN 4546 (paratype of C. cambara, Levi, 1986); 31-32 male MCN 52198. 28, dorsal; 29, ventral; 30, lateral view; 31, palp (ventral view); 32, paracymbium (ventral view). Scales bars: Figs 28-30, $1 \mathrm{~mm}$; Figs 31, $32,0.5 \mathrm{~mm}$.

\section{Chrysometa ludibunda (Keyserling, 1893)}

(Fig. 39)

Argyroepeira ludibunda KeyserLing, 1893:352, pl. 18, fig. 260. Female lectotype from Monte Verde and male paralectotype from Serra Vermelha, both Rio de Janeiro, Brazil deposited BMNH (not examined). Meta ludibunda; Petrunkevitch, 1911:362; Roewer, 1942:919; BonNET, 1957:2787.

Chrysometa ludibunda; LEVI, 1986:175, figs 491-496.
Other material examined. BRAZIL, Paraná: Guaraqueçaba*, đ, 20.IX.2007, E. Folly-Ramos leg. (MCN 44805); ㅇ; 18.IX.2007, G. Perroni leg. (MCN 45274); ㅇ, 18.IX.2007, F. Raub leg. (MCN 45928); Antonina*, J, 28.IX.2005, E. Alvarez leg. (MCN 44639); ㅇ, 21.IX.2005, E. Alvarez leg. (MCN 44762); ㅇ, 24.IX.2005, R. Ott leg. (MCN 45360); +, 26.IX.2005, F. Raub leg. (MCN 45621); Três Barras do Paraná*, ㅇ, 20-26.II.1993, A. B. Bonaldo leg. (MCN 23170); 2へ, 27.II.1993, A. B. Bonaldo leg. (MCN 23220); Capitão Leonidas Marques*, ô, 20-28. III.1993, A. B. Bonaldo leg. (MCN 23276); ㄱ, 4우 20-28.III.1993, A. B. Bonaldo leg. (MCN 23295); ㅇ, 20-28.III.1993, A. B. Bonaldo 


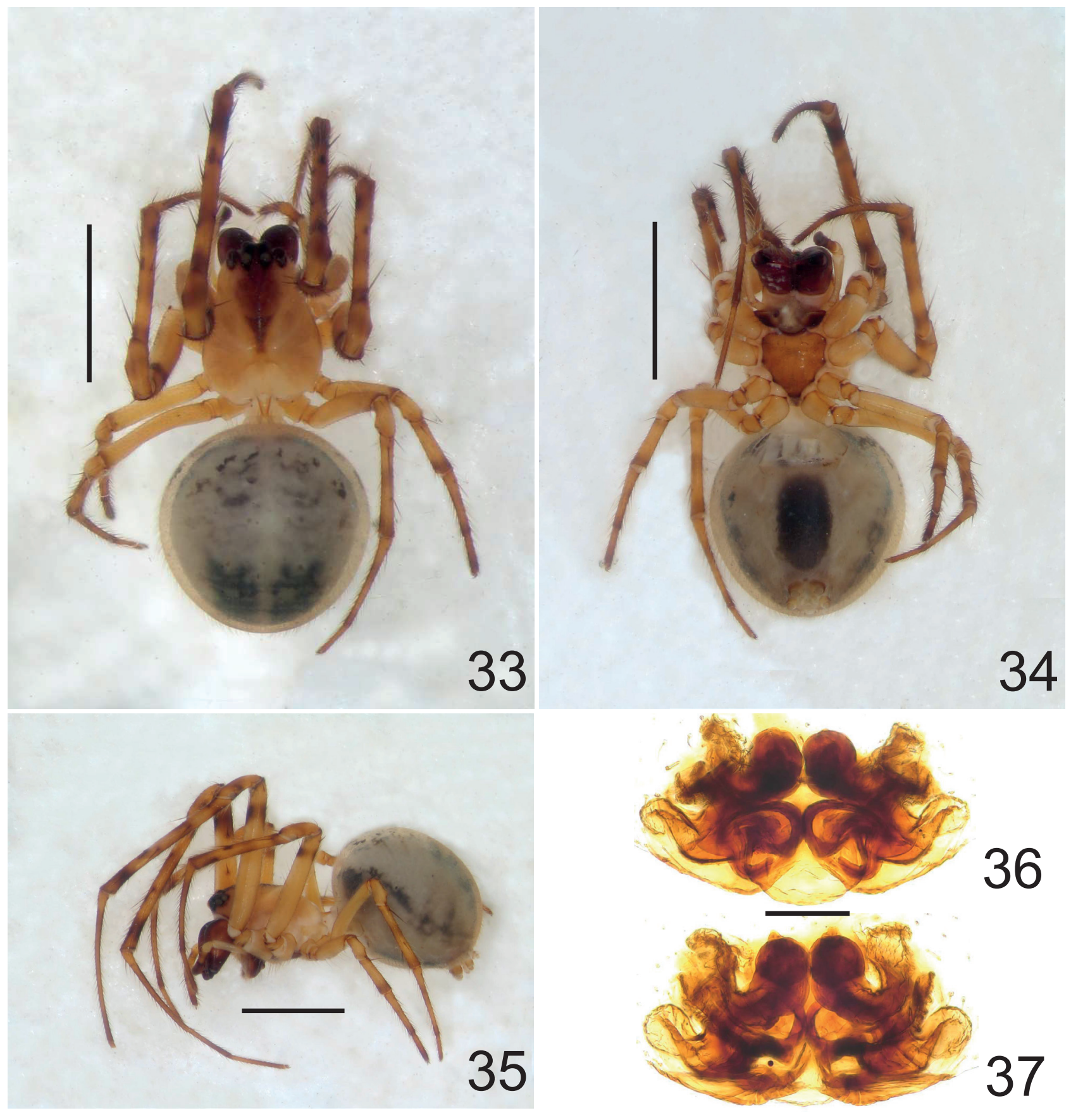

Figs 33-37. Chrysometa itaimba Levi, 1986. Female holotype MCN 12796. 33, dorsal; 34, ventral; 35, lateral; 36, epigyne (ventral view cleared); 37, epigyne (dorsal view cleared). Scales bars: Figs 33-35, 2 mm; Figs 36, 37, $0.2 \mathrm{~mm}$.

leg. (MCN 23477). Santa Catarina: Ilhota*, 2 , 13.V.1996, C. N. Duckett leg. (MCN 27632). Rio Grande do Sul: Derrubadas, $\partial$, 2731.X.2003 (MCN 37759); 60 6ㅇ, 27-31.X.2003 (MCN 38763); ð, ㅇ, 27-31.X.2003 (MCN 38773); 6ㅇ, 04-07.V.2004 (MCN 38781); ठำ

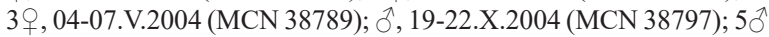
5ㅇ, 25-30.IV.2005 (MCN 39185), all R. Ott et al. leg.

\section{DISCUSSION}

The presented data allowed increasing the distribution data of Chrysometa aramba, C. boraceia, C. itaimba, C. ludibunda and especially $C$. itaimba. However the correction of the assigned males of C. cambara, which were considered here to belong to $C$. itaimba, changes considerably the range of $C$. cambara presented by BUCKUP et al. (2010). This species, which was formerly also assigned to Pampa areas of Rio Grande do Sul state, is now known only from the north half of the state, in the Araucarias plateau. On the other hand, the range of $C$. ludibunda, originally described from Rio de Janeiro state, is increased to Paraná and Santa Catarina states, being recorded in Rio Grande do Sul only at the very northern border of state. 

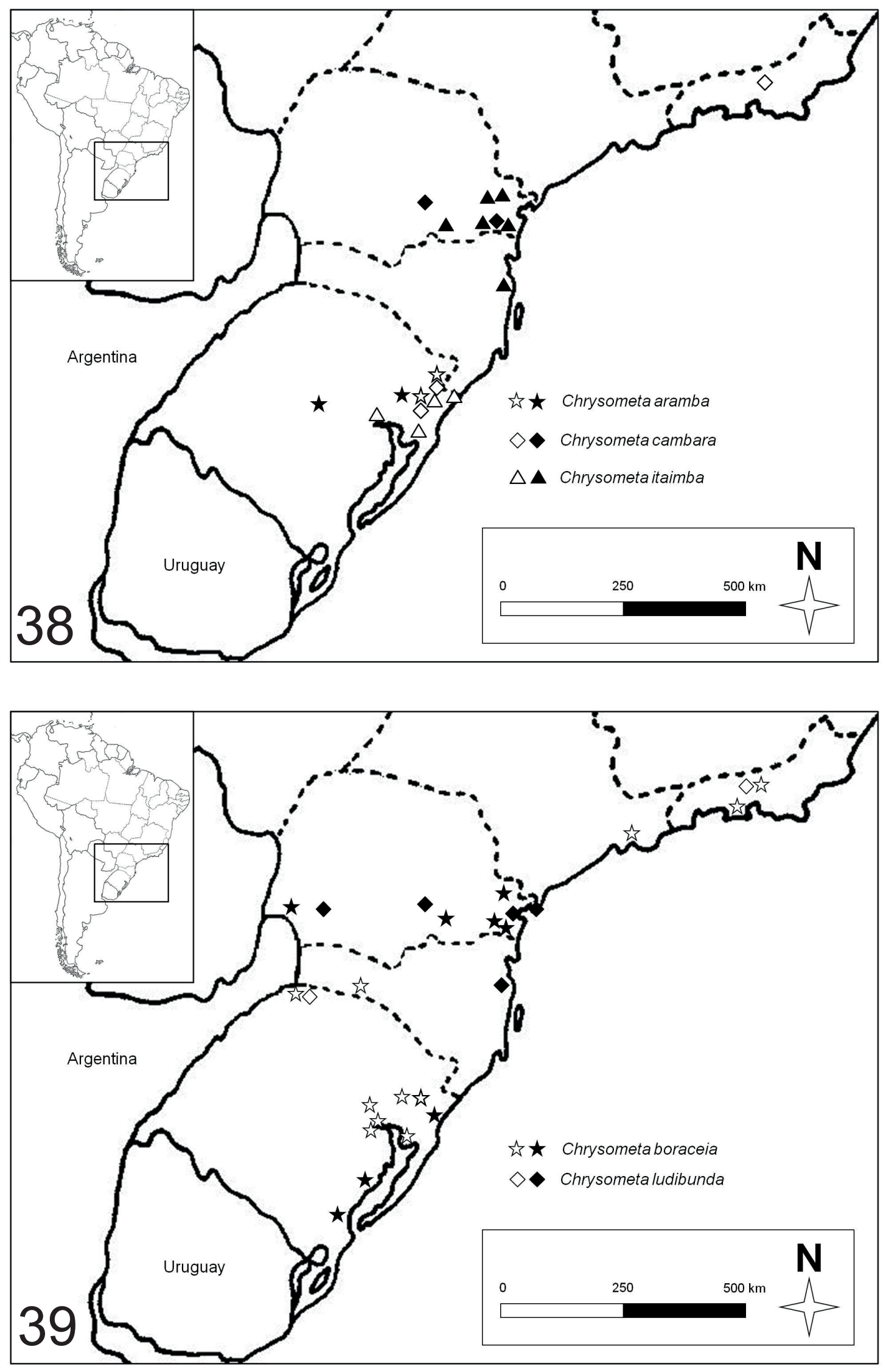

Figs 38, 39. 38, Meridional Brazil with records of Chysometa aramba, C. cambara and C. itaimba; 39, meridional Brazil with records of Chysometa boraceia and C. ludibunda. Markations indicate records of species, black filled ones indicate herein presented new records. 
Acknowledgements. To Creusa de Brobio and Janael Ricetti for all support. Suellen Breda for reviewing the text. To anonymous reviewers for the valuable suggestions on the manuscript.

\section{REFERENCES}

Álvarez-Padilla, F. 2007. Systematics of the spider genus Metabus O. P.-Cambridge, 1899 (Araneoidea: Tetragnathidae) with additions to the tetragnathid fauna of Chile and comments on the phylogeny of Tetragnathidae. Zoological Journal of the Linnean Society 151:285335.

Álvarez-Padilla, F.; Dimitrov, D.; Giribet, G. \& Hormiga G. 2009. Phylogenetic relationships of the spider family Tetragnathidae (Araneae, Araneoidea) based on morphological and DNA sequence data. Cladistics 25:109-146.

Álvarez-Padilla, F. \& Hormiga, G. 2011. Morphological and phylogenetic atlas of the orb-weaving spider family Tetragnathidae (Araneae: Araneoidea). Zoological Journal of the Linnean Society 162:713-879.

BonNet, P. 1957. Bibliographia araneorum. Toulouse, v. 2, n. 3, p. 1927-3026.

Buckup, E. H.; Marques, M. A. L.; Rodrigues, E. N. L. \& Ott, R. 2010. Lista das espécies de aranhas (Arachnida, Araneae) do estado do Rio
Grande do Sul, Brasil. Iheringia, Série Zoologia 100(4):483-518.

Keyserling, E. 1893. Die Spinnen Amerikas. Epeiridae. Nürnberg, v. 4, p. 209-377.

Kozub, D.; Khmelik, K.; Shapoval, J.; Chentsov, V.; Litovschenko, B. $\&$ StARIKH, V. 2012. Helicon Focus 5.3. Helicon Focus Ltda. Available at $<$ http://cybercom.net/ dcoffin/dcraw/>. Accessed on 23 May 2013.

Levi, H. W. 1986. The Neotropical orb-weaver genera Chrysometa and Homalometa (Araneae: Tetragnathidae). Bulletin of the Museum of Comparative Zoology 151:91-215.

Nogueira, A. A.; Pen-Barbosa, J. P. P.; Venticinque, E. M. \& Brescovit, A. D. 2011. The spider genus Chrysometa (Araneae, Tetragnathidae) from the Pico da Neblina and Serra do Tapirapecó mountains (Amazonas, Brazil): new species, new records, diversity and distribution along two altitudinal gradients. Zootaxa 2772:33-51.

Petrunkevitch, A. 1911. A synonymic index-catalogue of spiders of North, Central and South America with all adjacent islands, Greenland, Bermuda, West Indies, Terra del Fuego, Galapagos, etc. Bulletin of the American Museum of Natural History 29: 1-791.

Roewer, C. F. 1942. Katalog der Araneae von 1758 bis 1940. Bremen, v. 1, p. 1-1040.

SimON, E. 1894. Histoire Naturelle des Araignées. Paris, v. 1, p. 488-760. WORLD SPIDER CATALOG. 2016. Available at $<$ http://www.wsc.nmbe.ch/>. Accessed on 24 October 2017. 